OPEN ACCESS

Edited by:

Cheng Xue Helena Qin,

Baker Heart and Diabetes Institute,

Australia

Reviewed by:

Neil Macritchie,

University of Glasgow,

United Kingdom

Francesco Rossi,

University of Campania Luigi

Vanvitelli, Italy

*Correspondence:

Binghui Wang

bing.wang@monash.edu

Huimin Fan

Frankfan@tongji.edu.cn

tThese authors have contributed equally to this work

Specialty section: This article was submitted to Cardiovascular and Smooth

Muscle Pharmacology,

a section of the journal

Frontiers in Pharmacology

Received: 12 May 2019 Accepted: 04 September 2019

Published: 15 October 2019

Citation:

Liu J, Zhou X, Meng Q, Huang KW,

Liu J, Tie J, Zhuang R, Chen G,

Zhang Y, Wei L, Huang L, Li CG,

Wang B, Fan H and Liu Z (2019)

AFC1 Compound Attenuated MI/R-

Induced Ventricular Remodeling via Inhibiting PDGFR and STAT Pathway.

Front. Pharmacol. 10:1142.

doi: 10.3389/fphar.2019.01142

\section{AFC1 Compound Attenuated MI/R- Induced Ventricular Remodeling via Inhibiting PDGFR and STAT Pathway}

\author{
Jie Liu',2t, Xiaohui Zhou 1,3t, Qingshu Meng ${ }^{1,3+}$, Kevin W. Huang ${ }^{4}$, Jing Liu ${ }^{1,2}$, Jinjun Tie ${ }^{1,2}$, \\ Rulin Zhuang ${ }^{1,2}$, Guohan Chen ${ }^{2}$, Yuhui Zhang ${ }^{5}$, Lu Wei ${ }^{1,3}$, Li Huang $^{4}$, Chun Guang Li ${ }^{6}$, \\ Binghui Wang ${ }^{3,4 *}$, Huimin Fan ${ }^{1,2,3,7 *}$ and Zhongmin Liu ${ }^{1,2,3,7}$

\begin{abstract}
${ }^{1}$ Research Center for Translational Medicine, Shanghai East Hospital, Tongji University School of Medicine, Shanghai, Cardiovascular Research and Education in Therapeutics, Department of Epidemiology and Preventive Medicine, Monash University, Melbourne, VIC, Australia, ${ }^{5}$ Department of Ultrasound, Shanghai East Hospital, Tongji University School of Medicine, Shanghai, China, ${ }^{6}$ NICM Health Research Institute, Western Sydney University, Westmead, NSW, Australia,
\end{abstract} \\ China, ${ }^{2}$ Department of Cardiovascular and Thoracic Surgery, Shanghai East Hospital, Tongji University School of Medicine, \\ Shanghai, China, ${ }^{3}$ Shanghai Heart Failure Research Center, Shanghai East Hospital, Shanghai, China, ${ }^{4}$ Monash Centre of \\ ${ }^{7}$ Department of Heart Failure, Shanghai East Hospital, Tongji University School of Medicine, Shanghai, China
}

Background: Effective interventions to improve the outcome of patients subjected to myocardial ischemia reperfusion (MI/R) are urgent in clinical settings. Tanshinone IIA (TSA) is reported to attenuate myocardial injury and improve ventricular remodeling post MI/R. Here, we evaluated the efficacy of AFC1 compound that is similar to TSA structure in murine MI/R models. We found that AFC1 had a comparable effect of improving murine cardiac function after MI/R while it was superior to TSA in safety profile. Administration of AFC1 reduced reactive oxygen species $(\mathrm{ROS})$ production, inflammatory cells infiltration, and the expression of platelet derived growth factor receptors (PDGFR) in infarcted myocardium. Treatment with AFC1 also attenuated MI/R-induced cardiac remodeling and contributed to the recovery of cardiac function. Additionally, AFC1 reversed the elevation of PDGFR expression induced by PDGF-AB in both neonatal rat cardiomyocytes (NCMs) and neonatal rat cardiac fibroblasts (NCFs) and suppressed PDGF-AB induced NCM hypertrophy via STAT3 pathway and NCF collagen synthesis through p38-MAPK signaling in vitro. Similarly, AFC1 may contribute to the recovery of cardiac function in mice post MI/R via suppressing STAT signaling. Our results confirmed that AFC1 exerts anti-hypertrophic and anti-fibrotic effects against MI/R-induced cardiac remodeling, and suggest that AFC1 may have a promising potential in improving the outcome of patients who suffered from Ml/R.

Keywords: AFC1 compound, myocardial ischemia reperfusion, platelet derived growth factor, platelet derived growth factor receptor, ventricular remodeling

\section{INTRODUCTION}

Ischemic heart disease (IHD) has been a leading cause to high morbidity and mortality in developed countries with increasing incidence in developing countries (Chan et al., 2011). Although timely intervention can restore coronary flow, the reperfusion process triggers myocardium injury (Yellon and Hausenloy 2007), known as myocardial ischemia reperfusion (MI/R) injury, and cardiac remodeling, and subsequent heart failure (HF), which are the predominant contributors of death 
worldwide (Murdoch et al., 2006; Sun, 2009; Wang et al., 2015a). Therefore, effective prevention and treatment strategies to attenuate or reverse MI/R-induced remodeling is of great clinical value in IHD patients.

Recently, the protective role and relatively less adverse reaction of traditional Chinese medicine (TCM) in IHD have been highlighted. Danshen, the dry roots and rhizome of Salvia miltiorrhiza Bge., has been widely used either alone or in combination with other herbal ingredients for patients with IHD and other cardiovascular diseases in both China and other countries because of its efficacy in improving microcirculation and protecting against myocardial ischemia (Cheng, 2007). For example, results from meta-analysis demonstrated the potential benefits of compound Danshen dripping pill (CDDP) for treating coronary heart disease (Luo et al., 2015; Huang et al., 2016). However, the overall quality of the evidences in the systematic reviews was poor and high-quality evidence is warranted to support the clinical application of CDDP in treating IHD. Tanshinone IIA (TSA) is the most abundant and active diterpenoid quinone compound among lipophilic components extracted from Danshen (Wu et al., 2013). It is reported that TSA can attenuate myocardial injury and improve ventricular remodeling post MI/R via reducing reactive oxygen species (ROS) generation in mitochondria (Zhou et al., 2003; Jin and Li, 2013; Jin et al., 2013). However, in clinical settings, the efficacy of TSA is limited because of its lipid-soluble property, low bioavailability, and short half-life (Liu et al., 2013). Therefore, TSA modification targeting the above shortcomings is a promising strategy for its development in MI/R therapy.

Recently our team has investigated various compounds with similar core structure of TSA, including AFC1. In this study, we proved its potential role in cardiac protective effect against cardiac cell injury, hypertrophy, and fibrosis in vitro and in vivo. Thus, AFC1 compound may become a novel therapeutic pharmaceutical for patients subjected to MI/R. Accordingly it is important to study the effect of AFC1 in vivo to evaluate its efficacy and possible mechanism of actions.

Previous studies have demonstrated the importance of growth factors in IHD (Liu et al., 2014; Pello et al., 2015). High level of platelet derived growth factor (PDGF) in infarcted hearts contributed to myocardial inflammation and fibrosis in rats (Zhao et al., 2011). PDGF family is composed of four kinds of isoforms, $-A,-B,-C$, and $-D$, which comprised homodimers of PDGF-AA, $-\mathrm{BB},-\mathrm{CC}$, and $-\mathrm{DD}$ and heterodimer of PDGF-AB (Price et al., 2003). PDGF exerts its biological activities through two distinct subtypes of tyrosine kinase receptors, PDGF receptors (PDGFR)- $\alpha$ and $-\beta$ expressed on cardiomyocytes (Vantler et al., 2010). Excessive expression of PDGF could result in deposition of extracellular matrix and further induces cardiac remodeling (Vantler et al., 2010; Zhao et al., 2011). PDGF could induce $\mathrm{H}_{2} \mathrm{O}_{2}$ (kind of ROS) generation in mouse embryonic fibroblasts (MEFs) by binding PDGFR (Choi et al., 2005). On the other hand, the inhibition of PDGF/PDGFR pathway could attenuate the vascular remodeling via reducing the inflammatory response in the hypertensive rat with myocardial fibrosis (Fan et al., 2013). Therefore, PDGF/PDGFR may promote the development of cardiac remodeling after MI/R by mediating oxidative stress and inflammatory response.
Our present study demonstrated for the first time that treatment with AFC1 compound effectively attenuates MI/Rinduced cardiac remodeling, accompanied by decreased PDGFR expression, oxidative stress, and inflammatory response in hearts post MI/R. Moreover, AFC1 compound inhibited NCM hypertrophy and NCF collagen synthesis induced by PDGF-AB and contributed to the recovery of cardiac function post MI/R via regulating STAT3 pathway.

\section{MATERIALS AND METHODS}

\section{Animals}

Specific pathogen-free, male C57BL/6 mice (8-10 weeks) were purchased from Slac Laboratory Animal Co. Ltd (Shanghai, China). All experiments were conducted in accordance with protocols approved by the Institutional Animal Care and Use Committee of Tongji University.

\section{Establishment of Myocardial Ischemia Reperfusion Murine Models}

Echocardiography was performed before the establishment of MI/R models. Mice with EF above 50\% were included in the in vivo experiment. MI/R models were established as described previously (Pu et al., 2013). Regional ischemia was achieved by ligation of LAD using a 10-0 silk suture with a section of silica gel tube. Successful myocardial ischemia was achieved when the anterior wall of the left ventricular (LV) turned pale. After $30 \mathrm{~min}$ of ischemia, the ligation was relieved and the successful reperfusion was confirmed by epicardial hyperemia. TSA ( $5 \mathrm{mg} / \mathrm{kg}$ ) or AFC1 compound (7 or $14 \mathrm{mg} / \mathrm{kg}$ ) were intraperitoneally administrated daily for 1 week following MI/R. Mice with the heart exposed through left thoracic incision without ligation of left anterior descending coronary artery (LAD) were included in the sham group. Mice with $\mathrm{LAD}$ ligation for $30 \mathrm{~min}$ and then reperfusion for 2 weeks were randomly assigned to the following groups (5 mice in each group): Sham, MI/R, MI/R+TSA (5 mg/kg), MI/R+AFC1-L (7 $\mathrm{mg} / \mathrm{kg})$, and MI/R+AFC1-H (14 mg/kg). Each experiment was repeated at least three times. The AFC1 compound was synthesized by CG LI's lab at Western Sydney University.

\section{Echocardiography}

On day 14 post $\mathrm{MI} / \mathrm{R}$, the mice were anesthetized using isoflurane then M-mode echocardiography was performed in mice with echocardiographic imaging system (Visualsonics, Canada) equipped with a $15-\mathrm{MHz}$ linear transducer. Parameters of cardiac function were measured digitally on the M-mode tracings. All echocardiographic procedures were performed by a qualified investigator who was blinded to the grouping and treatment. The long-axis and short-axis view in B-mode were obtained. The B-mode guided M-mode view at the papillary muscle level was obtained for the evaluation of parameters. The end-systolic and end-diastolic LV dimensions were captured to calculate the LV ejection fraction (EF) and fractional shortening (FS) as previously described (Lin et al., 2013). 


\section{Histology}

After the reperfusion, fresh heart biopsies were fixed in $4 \%$ paraformaldehyde overnight at $4^{\circ} \mathrm{C}$ and embedded in paraffin. Sections into $5-\mu \mathrm{m}$ slices were stained with hematoxylin-eosin (H\&E) or Masson's trichrome for assessment of fibrosis. Tissues for immunofluorescence were submerged in liquid nitrogen and then embedded in optimal cutting temperature (OCT) solution (Sakura Finetek, USA) on dry ice to be frozen completely. Cardiomyocyte hypertrophy was examined in the peri-infarct zone. Myocyte cross-sectional areas were measured using Image J software (National Institutes of Health) in frozen sections stained with $5 \mu \mathrm{g} / \mathrm{ml}$ wheat germ agglutinin (WGA-Alexa Fluor $^{\circledR} 488$ conjugate, Invitrogen, USA). Five parts were chosen in the WGA images (200X) including left top, right top, middle, left bottom, and right bottom, and six cells were analyzed for each part. In other experiments, the hearts were excised for Masson staining to evaluate the cardiac remodeling. For inflammatory cell infiltration and PDGFR protein expression, immunofluorescence staining with anti-CD45 (Cell Signaling Technology, USA) and anti-PDGFRa (Cell Signaling Technology, USA) in frozen sections was conducted. Then the number of CD45+ cells/ field were quantified by Image J software (National Institutes of Health, USA).

\section{ROS Production}

ROS production was evaluated with dihydroethidium (DHE, Sigma, USA) on frozen myocardial sections. Heart slices were incubated at $37^{\circ} \mathrm{C}$ for $30 \mathrm{~min}$ with $10 \mu \mathrm{mol} / \mathrm{L}$ DHE in phosphatebuffered saline (PBS). Staining was captured by fluorescence microscope (Leica, Germany). Fluorescence intensity was quantified by using Image $J$ software (National Institutes of Health, USA).

\section{Isolation and Culture of Primary Cardiomyocytes and Cardiac Fibroblasts}

NCM and NCF were isolated from 1-day-old pups with enzymatic digestion as described previously (Thomas et al., 2002; Tzanidis et al., 2003). Purified NCMs were seeded at high density of $1 \times 10^{6}$ in 6 -well plate and $3 \times 10^{5}$ in 12 -well plate and maintained in serum-free DMEM (Gibco, USA) supplemented with $5 \mathrm{mg} / \mathrm{ml}$ insulin, $10 \mathrm{mg} / \mathrm{ml}$ apo-transferrin, and $50 \mathrm{mM}$ $\mathrm{KCl}$. Bromodeoxyuridine (0.1 mM, Sigma, USA) was applied for the first 3 days. NCF were seeded at a density of $3 \times 10^{5}$ in 6-well plate and $5 \times 10^{4}$ in 12 -well plate and cultured in DMEM HG supplemented with $0.5 \%$ BSA and $1 \% \mathrm{~L}$-ascorbic acid (Sigma, USA). On the fourth day, $1 \mathrm{~h}$ after pretreatment with AFC1 $(0.1,1.0,3.0,10.0 \mu \mathrm{M})$, PDGF-AB $(10 \mathrm{ng} / \mathrm{ml}$, PEPROTECH, USA) was added to induce hypertrophy in NCM and collagen synthesis in NCF. After $48 \mathrm{~h}$ of PDGF-AB stimulation, cells were harvested to determine hypertrophy and fibrosis, defined as a significant increase in protein content via ${ }^{3} \mathrm{H}$-Leucine or ${ }^{3} \mathrm{H}$-Proline incorporation. Cells were treated with AFC1 or DMSO for $1 \mathrm{~h}$ and then PDGF-AB for another 15 min for protein sample collection and Western blot detection.

\section{${ }^{3} \mathrm{H}$-Leucine and ${ }^{3} \mathrm{H}$-Proline Incorporation}

On the fourth day, after addition of PDGF-AB, NCMs were labeled with ${ }^{3} \mathrm{H}$-Leucine $(1 \mu \mathrm{Ci})$ and NCF with ${ }^{3} \mathrm{H}$-Proline $(5 \mu \mathrm{Ci})$ (PerkinElmer, USA) for $48 \mathrm{~h}$. The experiment was terminated by washing the cells with cold PBS for three times then precipitating with 10\% trichloroacetic acid (TCA, Sigma, USA) for $30 \mathrm{~min}$. Cells were then lysed in $1 \mathrm{M} \mathrm{NaOH}$ overnight in $4^{\circ} \mathrm{C}$. After neutralization with $1 \mathrm{M} \mathrm{HCl}$ and addition of scintillation fluid (PerkinElmer, USA), radioactivity was captured in a liquid scintillation counter (HIDEX 300 SL, Finland). The results represent at least three separate experiments done in triplicate for each condition.

\section{MTT Assay}

Cell viability was determined with colorimetric method using the MTT assay. NCFs were seeded at a density of $1.5 \times 10^{3}$ and human umbilical vein endothelial cells (HUVECs, PromoCell) at $1 \times 10^{4}$ cells per well in 96-well plate. After treatments for $48 \mathrm{~h}$, cells were incubated with $10 \mu \mathrm{l}$ of $5 \mathrm{mg} / \mathrm{ml}$ MTT (3-(4,5-dimethyl-2-thiazolyl)-2,5-diphenyl-2-H tetrazolium bromide (Sigma, USA) solution at $37^{\circ} \mathrm{C}$ for $4 \mathrm{~h}$. The formazan crystals were dissolved in $100 \mu \mathrm{l}$ of isopropanol for $20 \mathrm{~min}$ at $37^{\circ} \mathrm{C}$, and absorbance at $570 \mathrm{~nm}$ was detected on a Microplate Reader (SpectraMax, USA).

\section{Quantitative RT-PCR (q-PCR)}

Total RNA $(1 \mu \mathrm{g})$ extracted from myocardium, NCM, and NCF were reverse transcribed with PrimeScript RT reagent Kit with gDNA Eraser (TaKaRa, Japan), and q-PCR was performed on the 7900HT Fast Real Time PCR System (Applied Biosystems, UK) with the SYBR mastermix (Applied Biosystems, UK). All primer sequences were listed in Table 1.

Comparison of gene expression in different samples was calculated as follows. Each sample was related to an internal control gene (GAPDH). For example, Sample A was the control sample and Sample B was the treated one.

$\Delta \Delta \mathrm{Ct}=\left(\mathrm{Ct}\right.$ gene of interest $\left.-\mathrm{Ct}_{\mathrm{GAPDH}}\right)$ sample $\mathrm{B}-(\mathrm{Ct}$ gene of interest - $\left.\mathrm{Ct}_{\mathrm{GAPDH}}\right)$ sample A.

Finally, relative quantification of gene expression (Sample B) $=2^{-\Delta \Delta C t}$.

\section{Western Blot}

Protein was extracted from NCM, NCF, and homogenized myocardium tissue in lysis buffer. Protein lysate concentrations were determined via Pierce BCA Protein Assay Kit (Termo Scientific, USA). Equal amount of protein sample (20-30 $\mu \mathrm{g} /$ lane) from each group was subjected to $10 \%$ SDS-PAGE and transferred onto nitrocellulose membranes. After blocking with $5 \%$ bovine serum albumin (BSA), membranes were incubated overnight with primary antibodies $(1: 1,000$, Cell Signaling Technology, USA) against p-JAK2, p-STAT3, STAT3, p-p38, GAPDH, and pan-actin. On the second day, the membranes were incubated with fluorescent secondary antibody DyLight 800-Goat Anti-Rabbit IgG (H+L) (KPL, 
TABLE 1 | Quantitative polymerase chain reaction primers.

\begin{tabular}{|c|c|c|}
\hline Gene & Forward primer (5'-3') & Reverse primer (5'-3') \\
\hline mPDGF-A & GAGGAAGCCGAGATACCCC & TGCTGTGGATCTGACTTCGAG \\
\hline mPDGF-B & CATCCGCTCCTITGATGATCTT & GTGCTCGGGTCATGTTCAAGT \\
\hline mPDGFR- $\alpha$ & ACACGTTTGAGCTGTCAACC & CCCGACCACACAAGAACAGG \\
\hline mPDGFR- $\beta$ & TTCCAGGAGTGATACCAGCTT & AGGGGGCGTGATGACTAGG \\
\hline $\mathrm{mlL}-1 \beta$ & CGAGGCTAATAGGCTCATCT & GTTGGAAGCAGCCCTTCAT \\
\hline $\mathrm{mTNF}-\alpha$ & AGCCGATGGGTTGTACCTTGTCTA & TGAGATAGCAAATCGGCTGACGGT \\
\hline mlL-6 & TGATGCACTTGCAGAAAACA & ACCAGAGGAAATITTCAATAGGC \\
\hline mGAPDH & AACTTGGCATTGTGGAAGG & ACACATTGGGGGTAGGAACA \\
\hline rPDGF-A & TTCTTGATCTGGCCCCCAT & TTGACGCTGCTGGTGTTACAG \\
\hline rPDGF-B & GCAAGACGCGTACAGAGGTG & GAAGTTGGCATTGGTGCGA \\
\hline rPDGF-C & CAGCAAGTTGCAGCTCTCCA & GACAACTCTCTCATGCCGGG \\
\hline rPDGF-D & ATCGGGACACTITGCGACT & GTGCCTGTCACCCGAATGTT \\
\hline rPDGFR- $\alpha$ & GCTACACGTTTGAGCTGTCAAC & ATGGTGGTCATCCACAAGC \\
\hline rPDGFR- $\beta$ & TCTCTCATCATCCTCATCATGC & CCTTCCATCGGATCTCATAGC \\
\hline rANP & GAGGAGAAGATGCCGGTAG & CTAGAGAGGGAGCTAAGTG \\
\hline ro-SKA & GCATGCAGAAGGAGATCACA & CATAGCACGATGGTCGATTG \\
\hline $\mathrm{r} \beta \mathrm{MHC}$ & AGATCGAGGACCTGATGGTG & GATGCTCTTCCCAGTTGAGC \\
\hline rCol Type I & CATGTTCAGCTITGTGGACCT & GCAGCTGACTTCAGGGATGT \\
\hline rCol Type III & GGTCACTITCACTGGTTGACGA & TTGAATATCAAACACGCAAGGC \\
\hline rGAPDH & ACAAGATGGTGAAGGTCGGTG & AGAAGGCAGCCCTGGTAACC \\
\hline
\end{tabular}

USA). The membranes were scanned by ODYSSEY infrared imaging system (LI-COR Biosciences, USA). After incubation of phosphorylated proteins, we used stripping buffer (beyotime, China) to extract antibodies. Then we did the blocking and the following incubation procedures to obtain total protein quantification.

\section{Statistical Analysis}

Data, all presented as mean \pm SEM, were analyzed using SPSS software, version 11.0 (SPSS Inc., Chicago, IL, USA). For in vivo experiments, the Mann-Whitney U test was used for comparisons between different groups. One-way analysis of variance with a Bonferroni post hoc test was used for multiple comparisons. $\mathrm{P}<0.05$ was considered statistically significant.

\section{RESULTS}

\section{Safety Profile of AFC1 In Vivo}

To determine the safety of AFC1 in vivo, we evaluated the pathology of liver, kidney, spleen, and lung from mice administrated with AFC1 compound for 14 days. As shown by Figure 1A, there are no significant morphological changes in these organs. Besides, AFC1 administration did not change the body weight of mice on day 14 (Figure 1B). Previous study showed the cytotoxic effect of TSA on human umbilical vein endothelial cells (HUVECs) in a dose-dependent manner (Nizamutdinova et al., 2012). Then we treated the HUVECs with AFC1 or TSA. MTT data showed no difference in HUVECs viability between AFC1 and TSA group when both concentrations are 0.1, 1.0, and 3.0 $\mu \mathrm{M}$ (Figure 1C). However, AFC1 treated cells showed higher viability than the TSA group at $10 \mu \mathrm{M}(\mathrm{p}<0.05)$ (Figure 1C).

\section{Administration of AFC1 Contributed to the Recovery of Cardiac Function After MI/R in Murine Models}

Further cardiac function data showed that both high dose AFC1 (14 mg/kg) compound and TSA (5 mg/kg) significantly improved the EF and FS and systolic left ventricular interior diameters (LVIDs) in MI/R hearts (Figure 1D). However, there was no significance when AFC1 and TSA treated groups were compared. Then we assessed whether low $(7 \mathrm{mg} / \mathrm{kg})$ and high dose $(14 \mathrm{mg} / \mathrm{kg})$ of $\mathrm{AFC1}$ have equal protective role in murine MI/R models. As shown by Figure 1E, only high dose of AFC1 increased the EF as well as FS and decreased LVIDs compared to the MI/R group on day 14 post operation.

\section{AFC1 Compound Attenuated Mi/R- Induced Cardiac Remodeling}

Both dosages of AFC1 reduced the heart to body weight ratio (HW/BW) of mice effectively in comparison to MI/R group on day 14 ( $\mathrm{p}<0.0001)$ (Figure 2A). WGA staining data revealed significant myocyte hypertrophy in MI/R group compared to the sham $(\mathrm{p}<0.0001)$ and treatment with AFC1 greatly inhibited cardiac hypertrophy ( $\mathrm{p}<0.01$ and $\mathrm{p}<0.001$ ) (Figures 2B, C). Furthermore, AFC1 treated mice showed alleviated cardiac fibrosis compared to the mice without treatment on day 14 following surgery (Figures 2D, E).

\section{Antioxidant and Anti-Inflammatory Effects of AFC1 Compound on Infarcted Myocardium Following MI/R In Vivo}

It is well established that oxidative stress and inflammation response contribute to cardiac remodeling and dysfunction following $\mathrm{MI} / \mathrm{R}$. We then detected the production of ROS in 
A

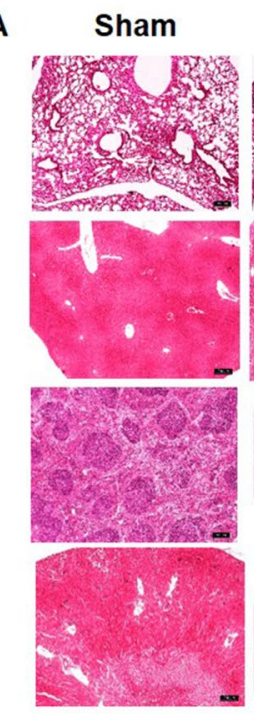

D

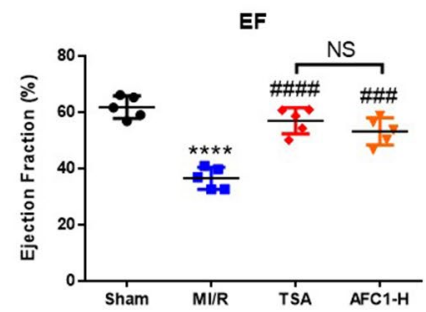

E

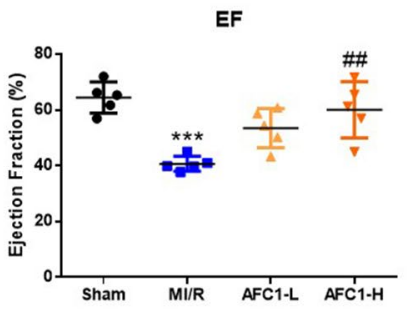

AFC1-H
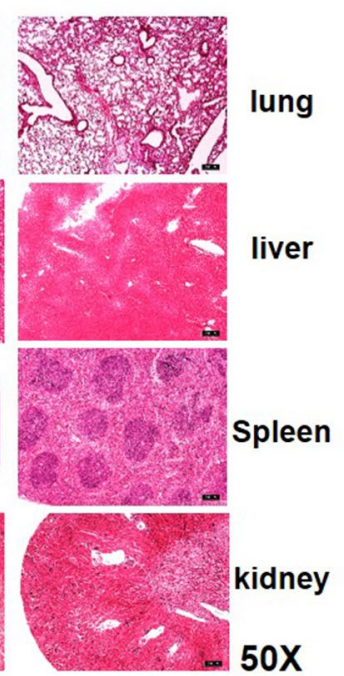

FS

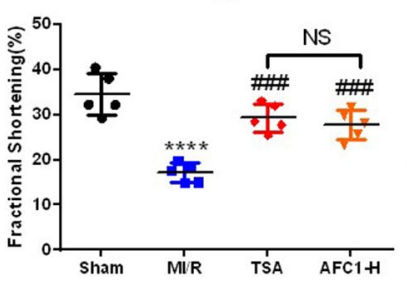

B

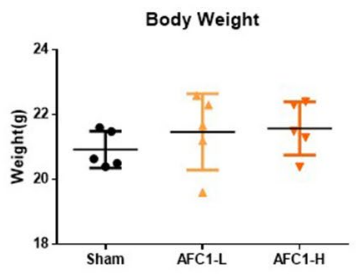

C

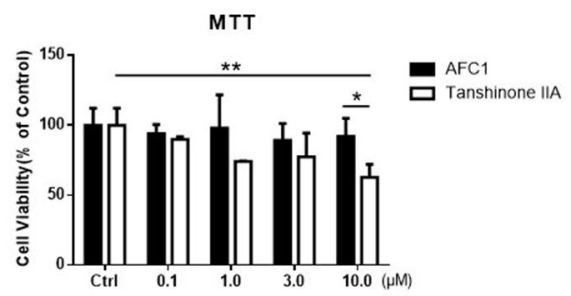

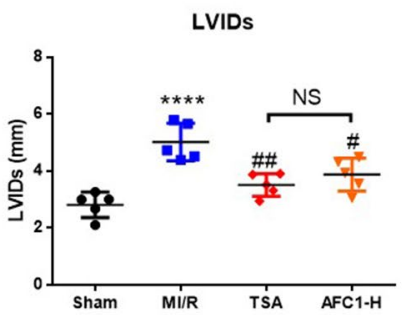

FS
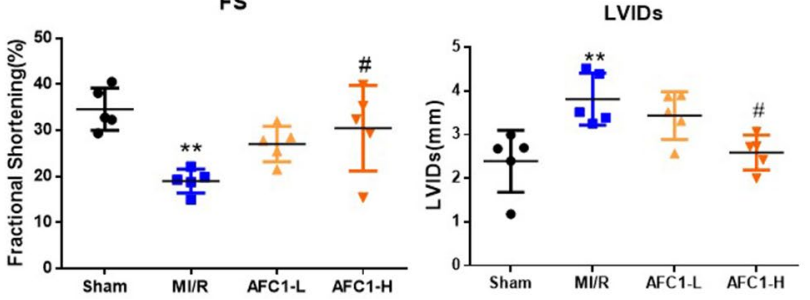

FIGURE 1 | Administration of AFC1 contributed to the recovery of cardiac function after MI/R in murine models. Mice were injected with low $(7 \mathrm{mg} / \mathrm{kg})$, high-dosage (14 mg/kg) of AFC1 compound or TSA (5 mg/kg) intraperitoneally for 7 days after MI/R $(n=5)$. (A) Lung, liver, spleen, and kidney sections were harvested on day 14 for H\&E staining. Figures showed the representative data. Magnification was $\times 50$. (B) Body weight change of mice after AFC1 administration was analyzed on day 14. (C) HUVECs were seeded at a concentration of $1 \times 10^{4} /$ well in 96-well plate and treated with DMSO, AFC1 compound, or TSA for $24 \mathrm{~h}$. Then cell viability was evaluated by MTT assay. (D) EF, FS, and LVIDs were measured by echocardiography on day 14 following MI/R. (E) Average values for EF, FS, and LVIDs. Each experiment was repeated for at least three times and results indicated mean \pm SEM of one independent experiment. ${ }^{*} p<0.05,{ }^{* \star} p<0.01,{ }^{* \star *} p<0.001$,

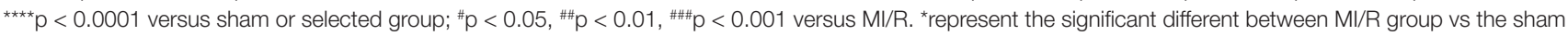
group; \#represent the significant different between treatment group and MI/R group.

hearts of different groups. MI/R significantly increased the ROS level, while AFC1 treatment greatly decreased ROS accumulation in the infarcted area $(\mathrm{p}<0.05)$ (Figures 3A, B). Figures 3C and D showed that high dosage of AFC1 dramatically decreased the CD45+ cell infiltrations in heart post MI/R ( $\mathrm{p}<0.0001)$. Moreover, both high and low dosages of AFC1 compound significantly down-regulated mRNA levels of inflammatory cytokines including IL-1 $\beta$, IL-6, and TNF- $\alpha$ in MI/R hearts (Figure 3E). These results suggested that AFC1 can attenuate MI/R-induced inflammatory responses in the heart.

\section{AFC1 Inhibited the Expression of PDGFR in Murine Heart Following MI/R}

Previous study revealed that PDGFs are involved in myocardial remodeling following infarction (Zhao et al., 2011). To verify whether MI/R could lead to an up-regulation of PDGF-related signaling, we measured the expression of PDGF-A, PDGF-B, and PDGFR isoforms in infarcted myocardium. As shown by Figure 4A, 30 min of ischemia followed by 2 weeks reperfusion markedly elevated mRNA levels of PDGFR $\alpha,-\beta$, PDGF-A, and PDGF-B (Figures 4A, B). Next, we treated the MI/R mice with AFC1 and data showed high dose of AFC1 down-regulated 


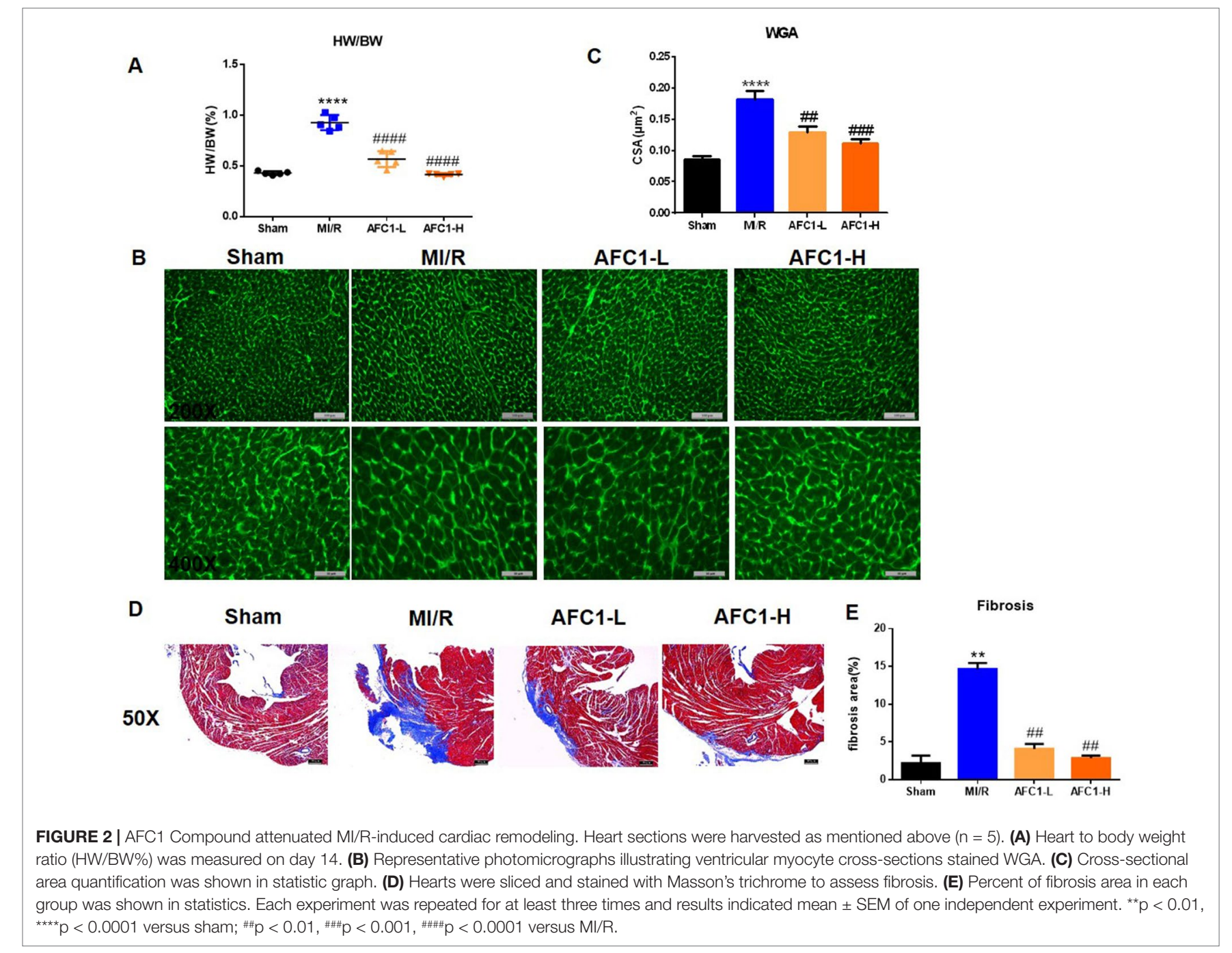

both PDGFR a and - $\beta$ mRNA levels in MI/R hearts. Low dose of AFC1 also decreased the expression of PDGFRa $(p<0.05)$. However, AFC1 did not affect PDGF-A or PDGF-B expression in hearts subjected to MI/R. Then we determined the PDGFRa protein level by immunofluorescence. As shown in Figure 4C, PDGFRa protein expression in the heart increased after MI/R, while it decreased after treatment with AFC1. (Nizamutdinova et al., 2012).

\section{Effect of PDGF Stimulation on PDGFR Expression in NCM and NCF In Vitro}

To further elucidate the role of PDGF signaling on heart remodeling, we treated NCM and NCF with different isoforms of PDGF for 48 or $72 \mathrm{~h}$ in vitro. As shown by Figure 5A, addition of PDGF-AA, PDGF-AB, PDGF-BB, or PDGF-CC all increased the mRNA levels of PDGFRa in NCM compared to the control media. Besides, PDGF-AB or PDGF-BB treatment up-regulated the PDGFR $\beta$ mRNA expression in NCM (Figure 5B). Figure 5C showed that NCM stimulated with PDGF-AB, PDGF-BB, or PDGF-CC for $48 \mathrm{~h}$ showed significant elevation of protein content in comparison to control cells. Moreover, administration of PDGF-AB or PDGF-BB triggered collagen synthesis in NCF (Figure 5D).

\section{AFC1 Reversed the Elevation of PDGFR Induced by PDGF-AB in Both NCM and NCF In Vitro}

Next, we treated NCM and NCF with PDGF-AB and AFC1 to determine whether AFC1 compound exerts its protective effect via regulating PDGF signaling in vitro. As shown by Figures 6A and $\mathbf{B}$, addition of AFC1 greatly decreased both PDGFRa and PDGFR $\beta$ mRNA expression in NCM stimulated with PDGF-AB. Moreover, high dose of AFC1 reversed the up-regulation of PDGFR $\beta$ mRNA levels induced by PDGF-AB in NCF (Figure 6C). MTT data (Figure 6D) showed that AFC1 did not affect the viabilities of NCF, further confirming that AFC1 inhibits the levels of PDGFR $\alpha$ and PDGFR $\beta$ stimulated by PDGF-AB in viable NCF cells. 


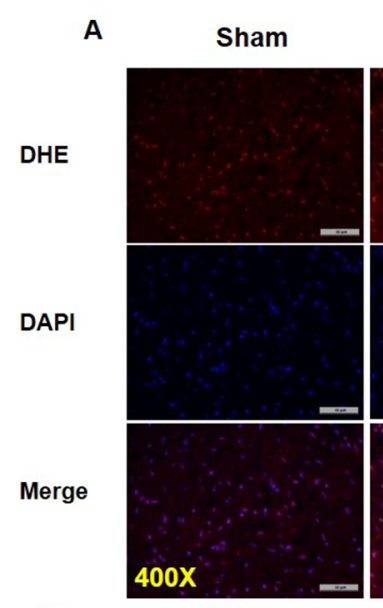

C
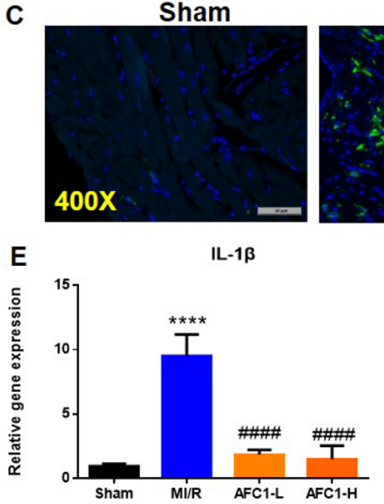

MI/R

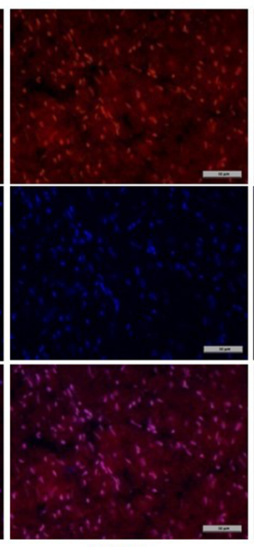

MI/R
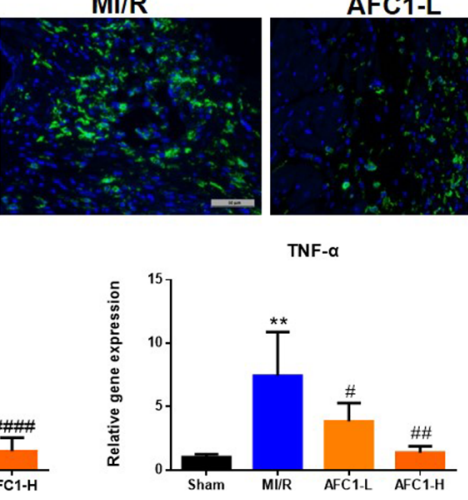

AFC1-L

AFC1-L
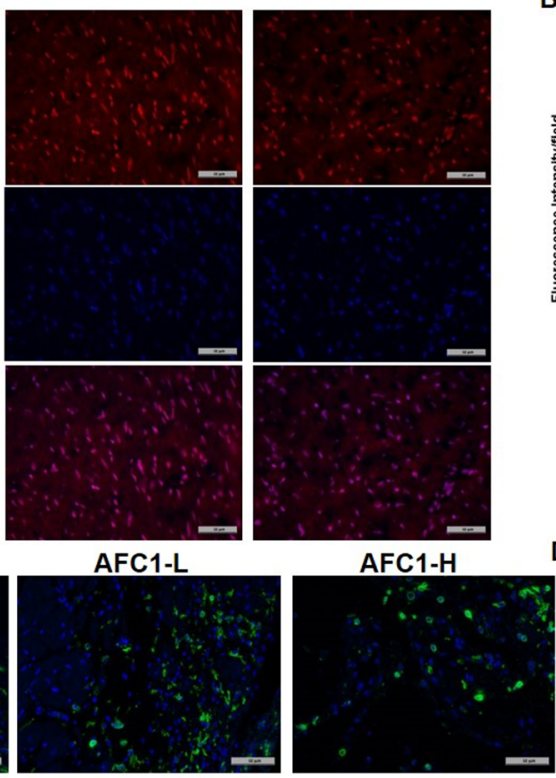

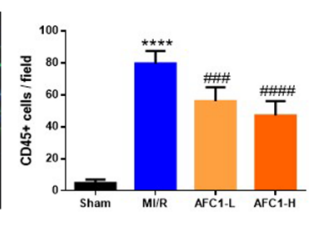

B

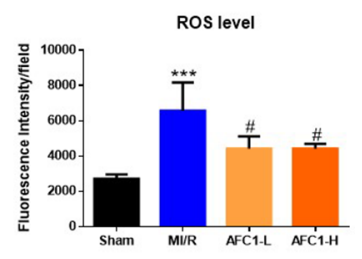

IL-6

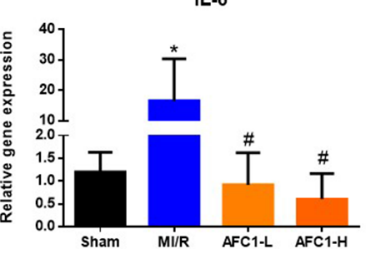

FIGURE 3 | Antioxidant and anti-inflammatory Effects of AFC1 compound on infarcted myocardium following MI/R in vivo. Heart sections were harvested and frozen as mentioned above $(n=5)$. (A) Reactive oxygen species production was assessed by dihydroethidium (DHE) conversion to red fluorescent ethidium. (B) Fluorescence intensity was evaluated to determine ROS level. (C) Immunofluorescence staining of CD45 in transverse section of heart (400x). (D) CD45+ cells were quantified in the field. (E) Hearts were homogenized and q-PCR was performed to quantify IL-1 $\beta$, TNF- $\alpha$, and IL- 6 mRNA levels. Results indicated mean \pm SEM of one representative experiment and the experiment was repeated three times. ${ }^{\star} p<0.05,{ }^{\star \star} p<0.01,{ }^{\star * \star} p<0.001,{ }^{\star \star \star \star *} p<0.0001$ versus sham; ${ }^{\#} p<0.05$, ${ }^{\# \#} p<0.01$, $\# \# p<0.001, \# \#$, $p<0.0001$ versus $M I / R$.

\section{AFC1 Suppressed NCM Hypertrophy and NCF Collagen Synthesis Induced by PDGF-AB}

To determine whether AFC1 compound can inhibit PDGF-AB induced NCM hypertrophy and NCF collagen synthesis, we examined the protein content via liquid scintillation detector to evaluate cardiac remodeling in vitro. Figure 7A showed that addition of AFC1 (1.0-10 $\mu \mathrm{M})$ significantly decreased NCM hypertrophy induced by PDGF-AB in a dose-dependent manner. Furthermore, AFC1 (3.0 and $10 \mu \mathrm{M})$ reversed the elevations of ANP, $\beta$-MHC, and $\alpha$-SKA mRNA levels stimulated by PDGF-AB in NCM (Figure 7B). AFC1 also inhibited the collagen synthesis (Figure 7C) as well as the mRNA expression of Col I and Col III in NCF stimulated by PDGF-AB (Figure 7D).

\section{AFC Modulated Signaling Pathways Involved in Cardiac Function and MI/R}

Previous reports revealed that STAT signaling pathway played a vital role in myocardial remodeling (Aboulhoda, 2017, Wincewicz and Sulkowski, 2017), and P38MAPK pathway is involved in cellular inflammatory response and apoptosis under the condition of ischemia and hypoxia (Owona et al., 2013). We cultured NCM and NCF with PDGF-AB with or without JAK inhibitor JI1 (JAKs inhibitor I-Calbiochem, Darmstadt, Germany) and p38 inhibitor 979 (Cai et al., 2018). As shown by Figures 8A and B, both $10 \mu \mathrm{M}$ JI1 and $3 \mu \mathrm{M} 979$ inhibited NCM hypertrophy and NCF collagen synthesis induced by PDGF-AB. Western blots analysis of protein expression of p-JAK2, p-STAT3, and p-p38 in cells revealed that PDGF-AB treatment greatly activated the phosphorylation of STAT3 in NCM and p-38 in NCF. Addition of AFC1 dramatically decreased the levels of p-STAT3 in NCM and p-p38 in NCF induced by PDGF-AB (Figures 8C, D). No significant difference was observed in the expression of p-JAK2 in these groups.

For in vivo study, MI/R increased the ratio of p-STAT3/GAPDH without affecting p-STAT3/STAT3 and STAT3/GAPDH in infarcted myocardium. Treatment with high dose of AFC1 markedly reversed the elevation of both p-STAT3/GAPDH and p-STAT3/STAT3 without affecting the ratio of STAT3/GAPDH (Figures 8E, F). 


\section{A}

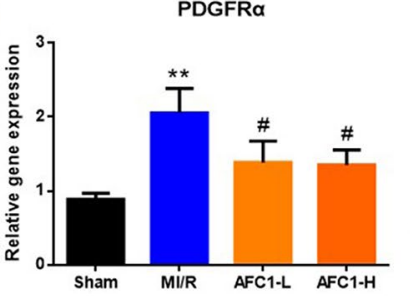

B

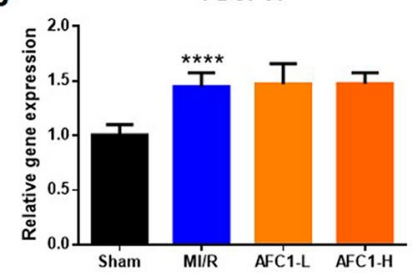

Sham

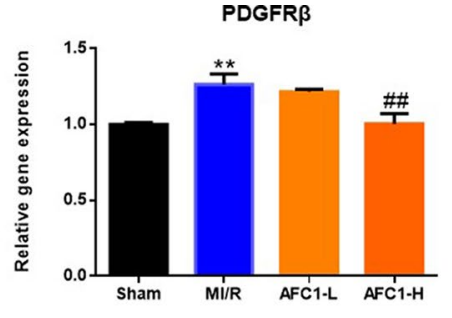

PDGF-B

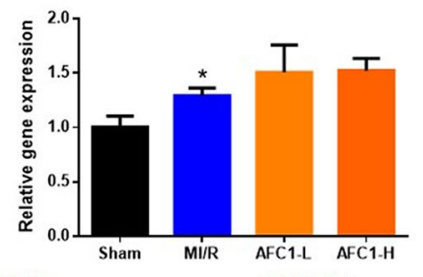

AFC1-L

AFC1-H
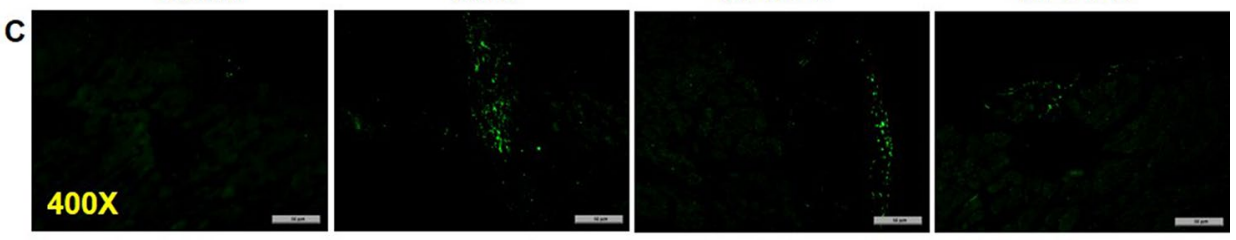

FIGURE 4 | AFC1 inhibited the expression of PDGFR in heart following MI/R $(n=5)$. Total RNA was extracted from hearts in each group and q-PCR was performed to quantify PDGFR $\alpha$, PDGFR $\beta$ (A), PDGF-A, and PDGF-B (B) mRNA levels. (C) Immunofluorescence staining of PDGFR $\alpha$ in transverse section of heart (400x). Each experiment was repeated for at least three times and results indicated mean \pm SEM of one representative experiment. ${ }^{*} p<0.05$, ${ }^{* *} p<0.01$, ${ }^{\star \star * *} p<0.0001$ versus sham; \#p $<0.05$, \#\# $<0.01$ versus Ml/R.

A

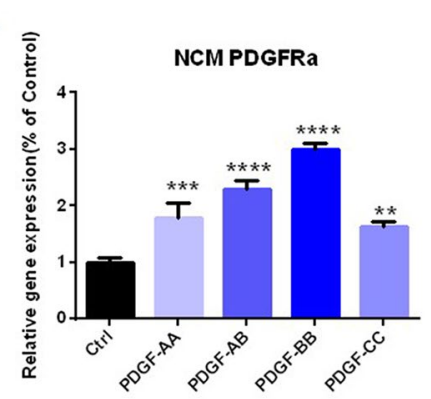

C

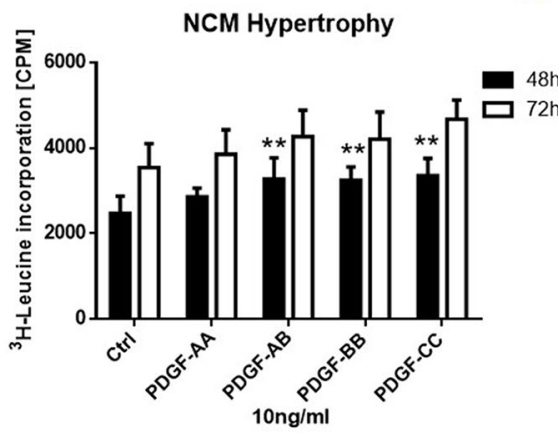

B

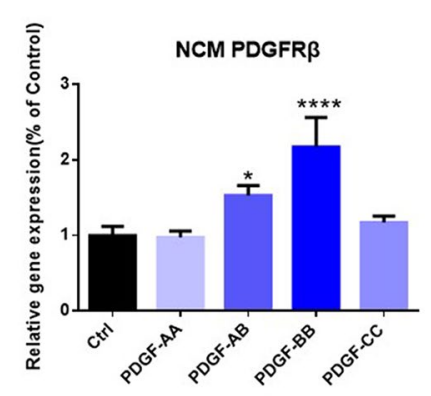

D



FIGURE 5 | Effect of PDGF stimulation on PDGFR expression in NCM and NCF in vitro. PDGFR $\alpha$ (A) and PDGFR 3 (B) mRNA expression in NCM were assessed by q-PCR. NCM hypertrophy (C) was measured by ${ }^{3} \mathrm{H}$-leucine incorporation and NCF collagen synthesis (D) measured by ${ }^{3} \mathrm{H}$-proline incorporation on different PDGF isoform stimulation. Each experiment was repeated for four times and results indicated mean $\pm \mathrm{SEM}$ of one representative experiment. ${ }^{*} \mathrm{p}<0.05$, ${ }^{\star \star} \mathrm{p}<0.01,{ }^{\star \star \star} \mathrm{p}<$ $0.001,{ }^{* \star \star *} \mathrm{p}<0.0001$ versus control. 
A

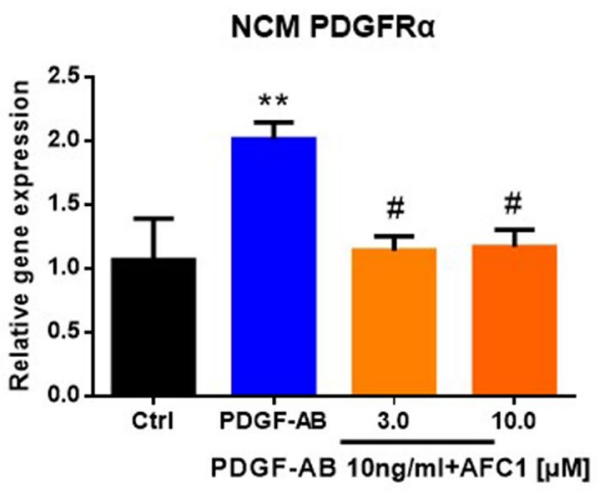

C

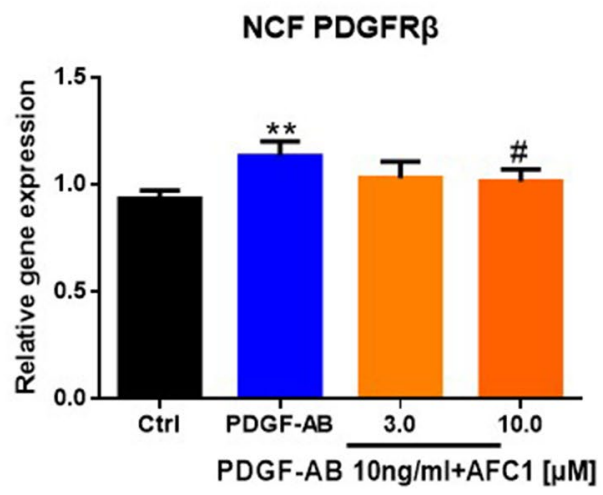

B

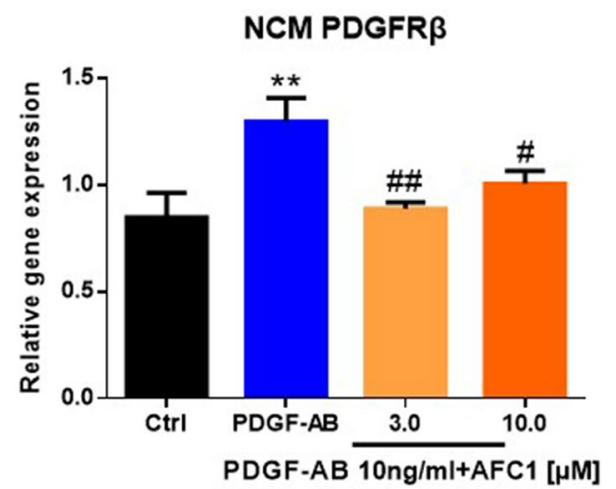

D

NCF MTT

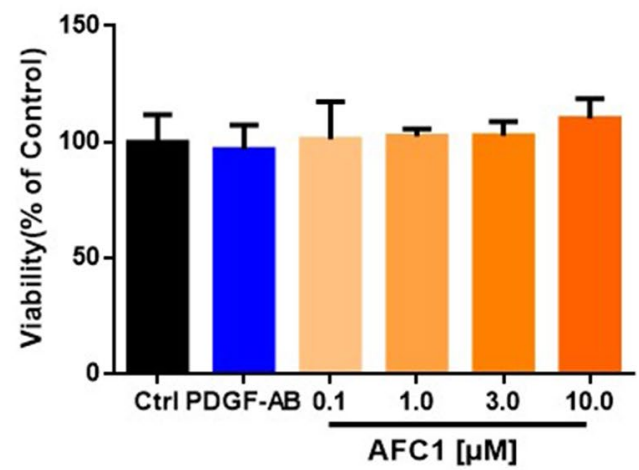

FIGURE 6 | AFC1 reversed the elevation of PDGFR induced by PDGF-AB in both NCM and NCF in vitro. PDGFR expression in NCM (A, B) and NCF (C) were quantified by q-PCR. (D) Viability of NCF stimulated with PDGF-AB or AFC1 compound (0.1, 1.0, 3.0, 10.0 $\mu \mathrm{M})$ was evaluated by MTT assay. This experiment was repeated four times and results indicated mean \pm SEM of one independent experiment and representative pictures. ${ }^{* *} p<0.01$ versus control; ${ }^{\# p}<0.05$, ${ }^{\#} p<0.01$ versus PDGF-AB.

A

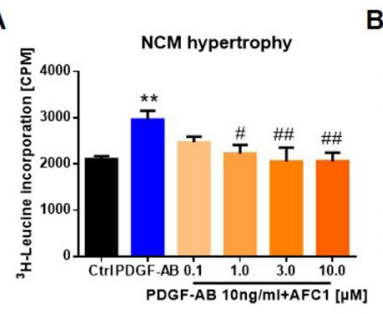

C

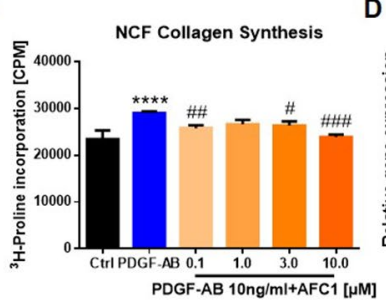

B

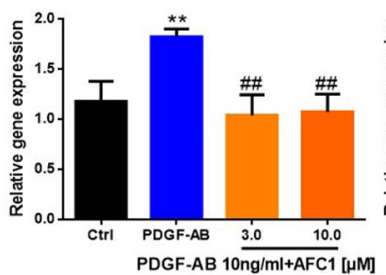

D

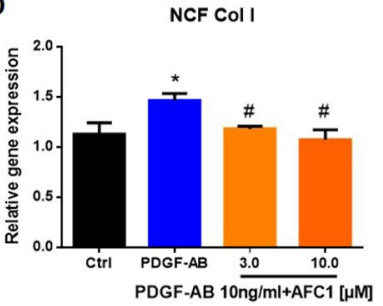

NCM $\beta-$ MHC

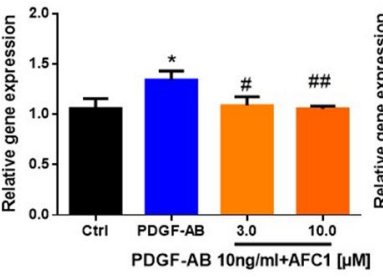

NCF Col III



FIGURE 7 | AFC1 suppressed PDGF-AB induced NCM hypertrophy and NCF collagen synthesis. NCM hypertrophy (A) was measured by ${ }^{3} \mathrm{H}$-leucine incorporation and NCF collagen synthesis (C) measured by ${ }^{3} \mathrm{H}$-proline incorporation on different concentration of AFC1 after PDGF-AB (10 ng/ml) stimulation. mRNA levels of ANP, $\beta-\mathrm{MHC}, \alpha-S K A$ in NCM (B), and Col I, Col III in NCF (D) were measured by q-PCR. This experiment was repeated four times and results indicated mean \pm SEM of one independent experiment. ${ }^{\star} p<0.05,{ }^{* \star} p<0.01,{ }^{* \star \star *} p<0.0001$ versus control; ${ }^{\#} p<0.05$, ${ }^{\# \#} p<0.01$, \#\#\# $<0.001$ versus PDGF-AB. 


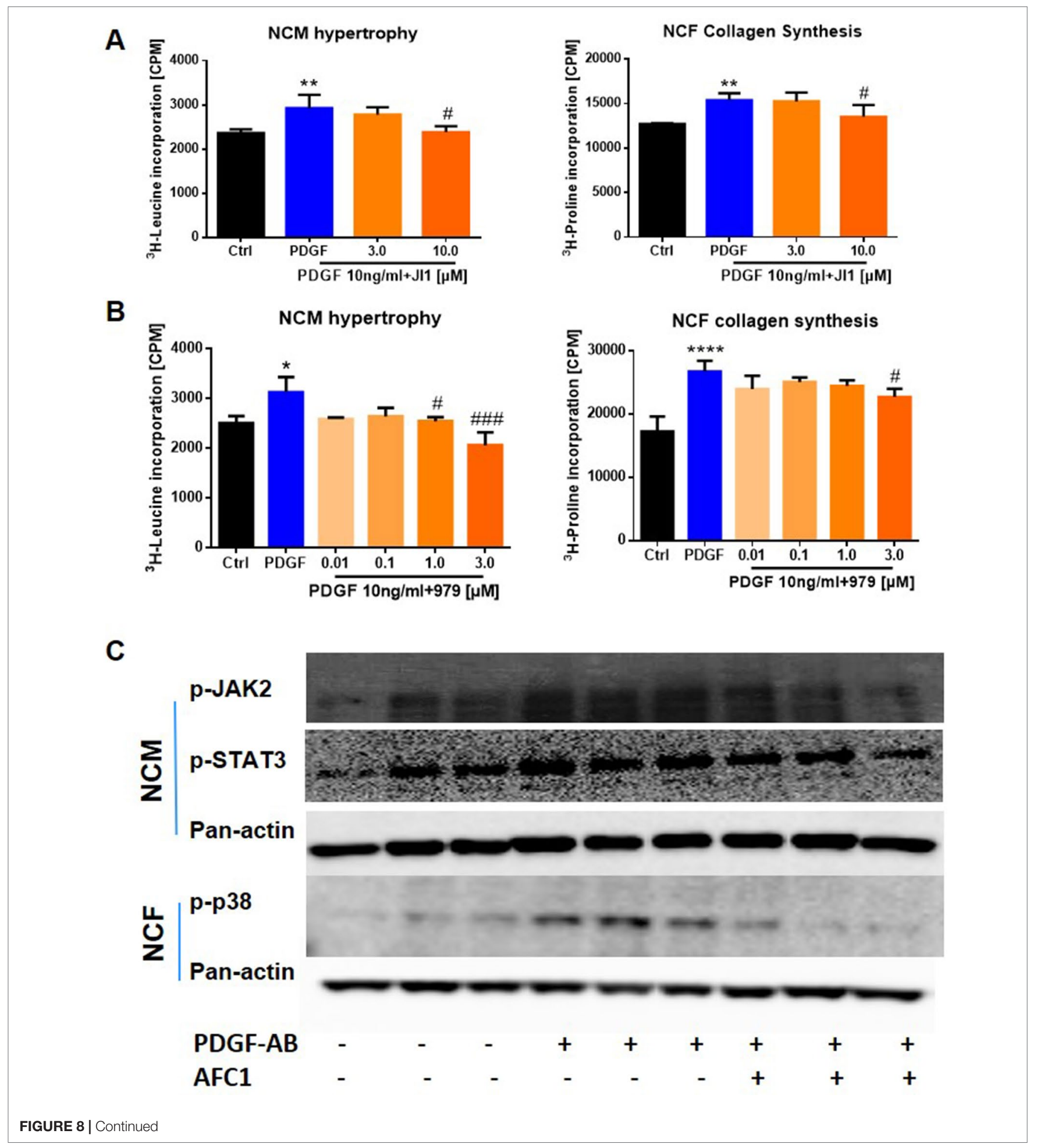

\section{DISCUSSION}

Early and effective intervention strategies have greatly decreased the mortality of IHD; however, reperfusion could cause myocardium damage, even exacerbate the cardiac function and structure (Wang et al., 2015b). Innovative pharmacotherapies to improve the outcomes especially left ventricular remodeling in patients who suffered from MI are still an urgent need (Della Rocca et al., 2012; Chew et al., 2018). Recently, TCMs have received much attention due to their functions in reduction of myocardial injury (Mo et al., 2015; Yang et al., 2016). Many studies demonstrated the cardioprotective effect of TSA and 


\section{D}

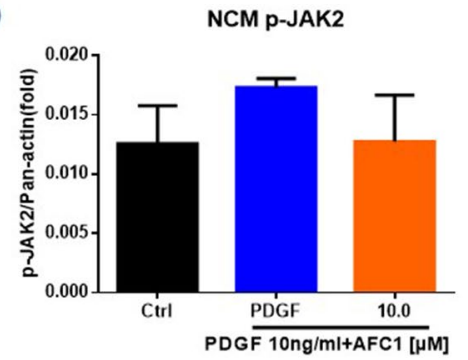

NCM p-STAT3

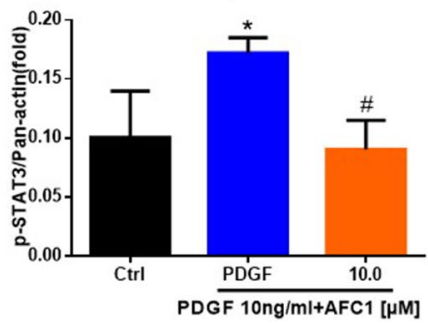

NCF p-p38

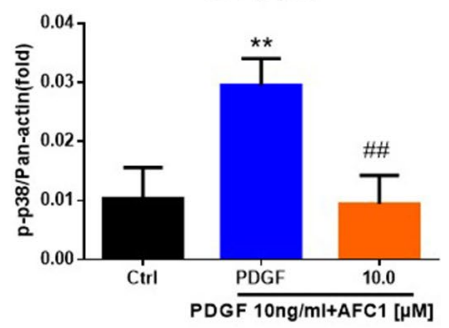

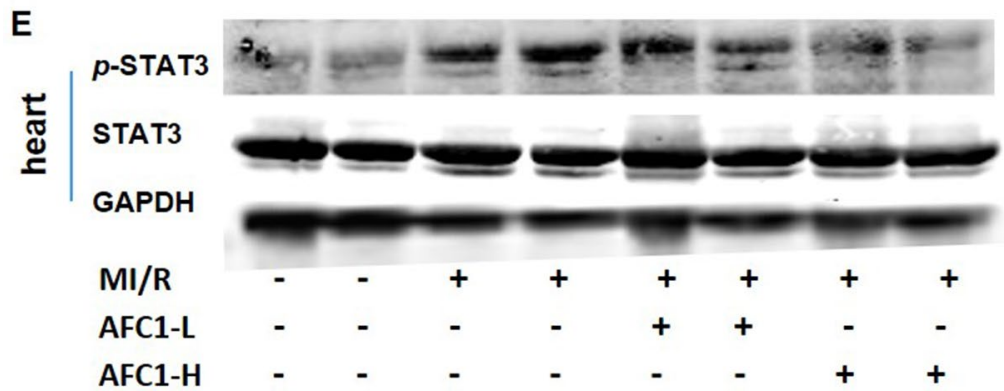

F
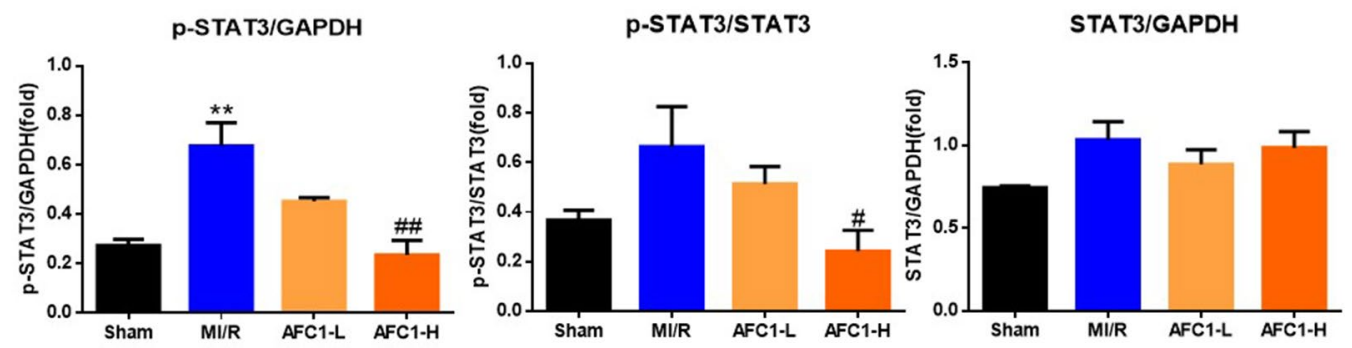

FIGURE 8 | Potential mechanism of the protective function of AFC1 treatment in vitro and in vivo $\mathrm{n}=5$. After being treated with $\mathrm{Jl} 1$ (A) or 979 (B) and then stimulated with PDGF-AB, NCM hypertrophy was measured by ${ }^{3} \mathrm{H}$-leucine incorporation and $\mathrm{NCF}$ collagen synthesis measured by ${ }^{3} \mathrm{H}$-proline incorporation. (C), D) Protein levels of p-JAK2, p-STAT3 in NCM and p-p38 in NCF were determined via Western blot analysis. (E) Infarct area of heart in each group was lysed and p-STAT3, STAT3 protein levels were determined via Western blot. (F) p-STAT3/STAT3, p-STAT3/GAPDH, and STAT3/GAPDH were analyzed with image J software. Two or three samples were randomly selected from each group for Western blot experiment. Each experiment was repeated for at least three times and results indicated mean \pm SEM of one independent experiment and the representative pictures. ${ }^{\star} p<0.05$, ${ }^{\star \star} p<0.01$, ${ }^{\star \star \star \star} p<0.0001$ versus control or sham; ${ }^{*} p<0.05$, $\# \mathrm{p}<0.01, \# \# \mathrm{p}<0.001$ versus PDGF-AB or $\mathrm{Ml} / \mathrm{R}$

sodium TSA sulfonate in myocardial ischemia/reperfusion injury animal models (Zhang et al., 2010; Zhang et al., 2013; Wei et al., 2014; Li Q et al., 2016; Pan et al., 2017). Results from the recent clinical trial indicated that sodium TSA sulfonate in combination with current therapies may significantly reduce adverse LV remodeling and potentially improve clinical outcomes, providing important evidence on the efficacy of sodium TSA sulfonate treatment in patients (Mao et al., 2015a; Mao et al., 2015b). Another trail named "Sodium Tanshinone IIA Sulfonate in Left Ventricular Remodeling Secondary to Acute Myocardial Infarction" is going on in Guangzhou, China (ClinicalTrials.gov Identifier: NCT02524964)(https://www.clinicaltrials.gov).

We have been searching new TCM-based cardioprotective agents and identified series AFC compounds with TSA mimic effects. In this study, we reported for the first time the cardioprotective actions of AFC1 in murine MI/R models. Ventricular remodeling including cardiac hypertrophy and fibrosis is the leading cause contributing to cardiac dysfunction after MI/R (Konishi et al., 2013). The key findings of the present study are that administration of AFC1 effectively reduced the cardiac myocyte hypertrophy and fibrosis on day 30 post MI/R, accompanied by significant improvement of cardiac function, and also inhibited MI/R-induced cardiac remodeling in vivo. The effect of AFC1 may be mediated by its inhibition of production of ROS and inflammation mediators and regulation of key signaling pathways, including PDGF, STAT3, and p38 signaling pathways.

$\mathrm{MI} / \mathrm{R}$ is a complex multifactorial pathophysiological process that oxidative stress and inflammatory response are key contributors to the following cardiac remodeling. The damage of oxygen free radical on vessel and myocardium after perfusion could result in myocardial injury and finally accelerate the development of MI/R (Duan et al., 2015). MI/R activated sterile inflammatory response characterized by the recruitment and activation of immune cells (Yan et al., 2013). Numerous studies have demonstrated that inhibition of neutrophil recruitment mediated by macrophage reduced tissue damage 
and infarct size in ischemic myocardium (Romson et al., 1983; de Lorgeril et al., 1989; Hatori et al., 1991; Li et al., 2016; Wang et al., 2016). Excessive neutrophil infiltration in the infarct site is detrimental to cardiomyocyte survival for secreting ROS, which further aggravates structural damage of tissue (Hansen, 1995). Our data showed that AFC1 compound inhibited the production of ROS, the infiltration of inflammatory cells, as well as the content of inflammatory cytokines, such as IL- $1 \beta$, TNF$\alpha$, and IL- 6 in infarcted myocardium, which implied that AFC1 compound may play an important role in improving ventricular remodeling after $\mathrm{MI} / \mathrm{R}$ via suppressing oxidative stress and inflammatory responses.

Previously, evidences indicated the crucial role of PDGF on stimulating fibrosis in many pathological conditions (Choi et al., 2005; Fan et al., 2013). Hypoxia could cause murine pulmonary vascular medial hypertrophy via increasing PDGF concentration (Zhang et al., 2012). PDGF-AB released by myofibroblast could cause myocyte structural and electromechanical remodeling in ovine persistent atrial fibrillation (PAF) through reducing calcium transients (Musa et al., 2013). The expression of PDGF is closely related to inflammation and fibrosis in infarcted myocardium (Zhao et al., 2011). In addition, PDGF can induce ROS production in MEFs (Choi et al., 2005). Therefore, PDGF may induce cardiac remodeling following MI/R via promoting oxidative stress and inflammatory response. The present result showed much higher expressions of PDGF-A, PDGF-B, PDGFR $\alpha$, and PDGFR $\beta$ in murine MI/R myocardium in MI/R heart than that in the sham group. Previous report from the MI rat models (Liu et al., 2014) revealed similar findings. Our results further indicated that AFC1 compound can effectively decrease the levels of PDGFR without affecting the expression of PDGF-A and PDGF-B in the heart post MI/R. Besides, AFC1 treatment greatly reduced PDGF-AB-stimulated PDGFR mRNA expression in NCM and NCF, as well as inhibited PDGF-ABstimulated NCM hypertrophy and NCF collagen synthesis in vitro. These data suggested that cardio-protective function of AFC1 may attribute to the inhibition of PDGFR signaling in vivo and in vitro.

It is proved that Janus kinase/signal transduction and activators of transcription (JAK/STAT) pathway can be activated by ischemic stress stimuli and cardiac hypertrophy agonist PDGF (Goodman et al., 2011; Wu et al., 2012). Tyrosine kinase could phosphorylate receptor tyrosine residues expressed on cardiomyocytes and activate the STAT phosphorylation. The activated STAT then transferred to nucleus and bonded to the target gene to regulate the expression of transcription factors or genes associated with hypertrophy and fibrosis, such as p21waf1 and c-fibrinogen (Wagner and Siddiqui, 2012). Inhibition of JAK/STAT pathway could reduce the myocardial infarct size and cardiomyocyte apoptosis induced by MI/R in rat models (Mascareno et al., 2001). Besides, PDGF-AB stimulated proliferation of human airway smooth muscle cells, which contribute to airway remodeling through the JAK/STAT pathway (Simon et al., 2002). Studies revealed that the PDGF/PDGFR pathway is involved in the regulation of cardiac function and the development of ventricular remodeling in MI/R via JAK/STAT downstream pathway (Wang et al., 2000; Booz et al., 2002). On the other hand, as an important intracellular signaling enzyme, P38MAPK is activated by myocardial ischemia and hypoxia to induce cellular apoptosis, and results in impaired cardiac function and amplifies the inflammatory cascade in the heart following MI/R. Our in vitro data showed that stimulation of PDGF-AB increased STAT3 phosphorylation in NCM and p38 phosphorylation in NCF, and addition of AFC1 compound significantly decreased both proteins' phosphorylation, as well as suppressed NCM hypertrophy and NCF collagen synthesis. Moreover, in murine MI/R models, the expression of p-STAT3 up-regulated in murine infarcted myocardium and this elevation can be dramatically decreased by AFC1 treatment, indicating the possible downstream pathway in which AFC1 exerts its role in cardiac myocyte. We therefore proposed that AFC1 compound may attenuate MI/R-induced cardiac remodeling via regulating PDGFR signaling and inhibiting the phosphorylation of STAT3. It is also possible that AFC1 may act on other singling pathways, but further study is needed to elucidate this.

Importantly, AFC1 showed neither detrimental impact on morphological and histological changes of murine lung, liver, kidney, and spleen, nor the cytotoxicity in HUVEC viability. It showed less cytotoxicity than TSA in high doses, indicating it may have a better safety profile than TSA. Since TSA has been widely used clinically with excellent safety profile, AFC1 may also be a potential clinical agent for treating MI/R. But further study is needed on its pharmacokinetic profile and more detailed evaluation of its toxicity in vivo. In addition, further investigations are needed to explore the role of AFC1 in other cardiovascular diseases and further in clinical trials.

\section{CONCLUSIONS}

AFC1 compound had comparable effect with TSA in improving cardiac function after MI/R. Administration of AFC1 suppressed STAT signaling and attenuated MI/R-induced cardiac remodeling in murine $\mathrm{MI} / \mathrm{R}$ models. AFC1 suppressed PDGF-AB induced NCM hypertrophy via STAT3 pathway and NCF collagen synthesis through p38 signaling. Therefore, AFC1 may be a novel therapeutic option with anti-hypertrophic and anti-fibrotic effect against MI/R-induced cardiac remodeling in patients who suffered from $M I / R$.

\section{DATA AVAILABILITY STATEMENT}

The raw data supporting the conclusions of this manuscript will be made available by the authors, without undue reservation, to any qualified researcher.

\section{ETHICS STATEMENT}

This study was carried out in accordance with the recommendations of the Institutional Animal Care and Use Committee of Tongji University. The protocol was approved 
by the Institutional Animal Care and Use Committee of Tongji University.

\section{AUTHOR CONTRIBUTIONS}

$\mathrm{XZ}, \mathrm{BW}$, and HF contributed to the conception and design of the study. JieL, XZ, QM, KH, JingL, JT, RZ, GC, YZ, LW, and LH contributed to acquisition, analysis, and interpretation of the data. JieL, XZ, CL, ZL, and BW wrote and revised the MS.

\section{REFERENCES}

Aboulhoda, B. E. (2017). Age-related remodeling of the JAK/STAT/SOCS signaling pathway and associated myocardial changes: from histological to molecular level. Ann. Anat. 214, 21-30. doi: 10.1016/j.aanat.2017.07.003

Booz, G. W., Day, J. N., and Baker, K. M. (2002). Interplay between the cardiac renin angiotensin system and JAK-STAT signaling: role in cardiac hypertrophy, ischemia/reperfusion dysfunction, and heart failure. J. Mol. Cell Cardiol. 34 (11), 1443-1453. doi: 10.1006/jmcc.2002.2076

Cai, Y., Lu, C., Xu, T., Ma, Y., Min, S., Scammells, P., et al. (2018). Diffusion tensor imaging evaluation of axonal/white matter remodeling in a mouse model of diabetic stroke treated with novel p38 MAPK inhibitor, VCP979. J. Biomed. Nanotechnol. 14 (3), 585-593. doi: 10.1166/jbn.2018.2522

Chan, E., Liu, X. X., Guo, D. J., Kwan, Y. W., Leung, G. P., Lee, S. M., et al. (2011). Extract of Scutellaria baicalensis Georgi root exerts protection against myocardial ischemia-reperfusion injury in rats. Am. J. Chin. Med. 39 (4), 693704. doi: 10.1142/S0192415X11009135

Cheng, T. O. (2007). Cardiovascular effects of Danshen. Int. J. Cardiol. 121 (1), 9-22. doi: 10.1016/j.ijcard.2007.01.004

Chew, D. S., Heikki, H., Schmidt, G., Kavanagh, K. M., Dommasch, M., Bloch Thomsen, P. E., et al. (2018). Change in left ventricular ejection fraction following first myocardial infarction and outcome. JACC Clin. Electrophysiol. 4 (5), 672-682. doi: 10.1016/j.jacep.2017.12.015

Choi, M. H., Lee, I. K., Kim, G. W., Kim, B. U., Han, Y. H., Yu, D. Y., et al. (2005). Regulation of PDGF signalling and vascular remodelling by peroxiredoxin II. Nature 435 (7040), 347-353. doi: 10.1038/nature03587

de Lorgeril, M., Basmadjian, A., Lavallee, M., Clement, R., Millette, D., Rousseau, G., et al. (1989). Influence of leukopenia on collateral flow, reperfusion flow, reflow ventricular fibrillation, and infarct size in dogs. Am. Heart J. 117 (3), 523-532. doi: 10.1016/0002-8703(89)90724-2

Della Rocca, D. G., Willenberg, B. J., Ferreira, L. F., Wate, P. S., Petersen, J. W., Handberg, E. M., et al. (2012). A degradable, bioactive, gelatinized alginate hydrogel to improve stem cell/growth factor delivery and facilitate healing after myocardial infarction. Med. Hypotheses 79 (5), 673-677. doi: 10.1016/j. mehy.2012.08.006

Duan, Z. Z., Li, Y. H., Li, Y. Y., Fan, G. W., Chang, Y. X., Yu, B., et al. (2015). Danhong injection protects cardiomyocytes against hypoxia/reoxygenationand $\mathrm{H} 2 \mathrm{O} 2$-induced injury by inhibiting mitochondrial permeability transition pore opening. J. Ethnopharmacol. 175, 617-625. doi: 10.1016/j.jep.2015.08.033

Fan, B., Ma, L., Li, Q., Wang, L., Zhou, J., and Wu, J. (2013). Correlation between platelet-derived growth factor signaling pathway and inflammation in desoxycorticosterone-induced salt-sensitive hypertensive rats with myocardial fibrosis. Int. J. Clin. Exp. Pathol. 6 (11), 2468-2475.

Goodman, M. D., Koch, S. E., Afzal, M. R., and Butler, K. L. (2011). STAT subtype specificity and ischemic preconditioning in mice: is STAT-3 enough? Am. J. Physiol. Heart Circ. Physiol. 300 (2), H522-H526. doi: 10.1152/ ajpheart.00231.2010

Hansen, P. R. (1995). Role of neutrophils in myocardial ischemia and reperfusion. Circulation 91 (6), 1872-1885. doi: 10.1161/01.CIR.91.6.1872

Hatori, N., Roberts, R. L., Tadokoro, H., Ryden, L., Satomura, K., Fishbein, M. C., et al. (1991). Differences in infarct size with lidocaine as compared with bretylium tosylate in acute myocardial ischemia and reperfusion in pigs. J. Cardiovasc. Pharmacol. 18 (4), 581-588. doi: 10.1097/00005344-199110000-00015

\section{FUNDING}

The study was supported by the National Key Research and Development Program of China (2017YFA0105600), the National Natural Science Foundation of China (81370434; $81670458 ; 81470393)$, the Shanghai Municipal Health and Family Planning Commission (ZY3-LCPT-2-1003-2014ZYJB0502), Key Discipline Project of Pudong Health Bureau of Shanghai (PWZxk2017-01), and the Science and Technology Commission of Shanghai Municipality (17431906600).

Huang, J., Tang, X., Ye, F., He, J., and Kong, X. (2016). Clinical therapeutic effects of aspirin in combination with Fufang Danshen Diwan, a traditional Chinese medicine formula, on coronary heart disease: a systematic review and metaanalysis. Cell Physiol. Biochem. 39 (5), 1955-1963. doi: 10.1159/000447892

Jin, H. J., and Li, C. G. (2013). Tanshinone IIA and cryptotanshinone prevent mitochondrial dysfunction in hypoxia-induced $\mathrm{H} 9 \mathrm{c} 2$ cells: association to mitochondrial ROS, intracellular nitric oxide, and calcium levels. Evid. Based Complement Alternat. Med. 2013, 610694. doi: 10.1155/2013/610694

Jin, H. J., Xie, X. L., Ye, J. M., and Li, C. G. (2013). TanshinoneIIA and cryptotanshinone protect against hypoxia-induced mitochondrial apoptosis in H9c2 cells. PLoS ONE 8 (1), e51720. doi: 10.1371/journal.pone.0051720

Konishi, K., Dohi, K., Tanimura, M., Sato, Y., Watanabe, K., Sugiura, E., et al. (2013). Quantifying longitudinal right ventricular dysfunction in patients with old myocardial infarction by using speckle-tracking strain echocardiography. Cardiovasc. Ultrasound 11, 23. doi: 10.1186/1476-7120-11-23

Li, Q., Shen, L., Wang, Z., Jiang, H. P., and Liu, L. X. (2016). Tanshinone IIA protects against myocardial ischemia reperfusion injury by activating the PI3K/Akt/mTOR signaling pathway. Biomed. Pharmacother. 84, 106-114. doi: 10.1016/j.biopha.2016.09.014

Li, W., Hsiao, H. M., Higashikubo, R., Saunders, B. T., Bharat, A., Goldstein, D. R., et al. (2016). Heart-resident CCR2+ macrophages promote neutrophil extravasation through TLR9/MyD88/CXCL5 signaling. JCI Insight 1 (12), E87135. doi: 10.1172/jci.insight.87315

Lin, S. Q., Wei, X. H., Huang, P., Liu, Y. Y., Zhao, N., Li, Q., et al. (2013). QiShenYiQi Pills(R) prevent cardiac ischemia-reperfusion injury via energy modulation. Int. J. Cardiol. 168 (2), 967-974. doi: 10.1016/j.ijcard.2012.10.042

Liu, C., Zhao, W., Meng, W., Zhao, T., Chen, Y., Ahokas, R. A., et al. (2014). Plateletderived growth factor blockade on cardiac remodeling following infarction. Mol. Cell Biochem. 397 (1-2), 295-304. doi: 10.1007/s11010-014-2197-x

Liu, X., An, C., Jin, P., Liu, X., and Wang, L. (2013). Protective effects of cationic bovine serum albumin-conjugated PEGylated tanshinone IIA nanoparticles on cerebral ischemia. Biomaterials 34 (3), 817-830. doi: 10.1016/j. biomaterials.2012.10.017

Luo, J., Song, W., Yang, G., Xu, H., and Chen, K. (2015). Compound Danshen (Salvia miltiorrhiza) dripping pill for coronary heart disease: an overview of systematic reviews. Am. J. Chin. Med. 43 (1), 25-43. doi: 10.1142/ S0192415X15500020

Mao, S., Li, X., Wang, L., Yang, P. C., and Zhang, M. (2015a). Rationale and design of sodium Tanshinone IIA sulfonate in left ventricular remodeling secondary to acute myocardial infarction (STAMP-REMODELING) trial: a randomized controlled study. Cardiovasc. Drugs Ther. 29 (6), 535-542. doi: 10.1007/ s10557-015-6625-2

Mao, S., Wang, L., Zhao, X., Shang, H., Zhang, M., and Hinek, A. (2015b). Sodium tanshinone IIA sulfonate for reduction of periprocedural myocardial injury during percutaneous coronary intervention (STAMP trial): rationale and design. Int. J. Cardiol. 182, 329-333. doi: 10.1016/j.ijcard.2014.12.166

Mascareno, E., El-Shafei, M., Maulik, N., Sato, M., Guo, Y., Das, D. K., et al. (2001). JAK/STAT signaling is associated with cardiac dysfunction during ischemia and reperfusion. Circulation 104 (3), 325-329. doi: 10.1161/01.CIR.104.3.325

Mo, W. L., Chai, C. Z., Kou, J. P., Yan, Y. Q., and Yu, B. Y. (2015). Sheng-Mai-San attenuates contractile dysfunction and structural damage induced by chronic intermittent hypoxia in mice. Chin. J. Nat. Med. 13 (10), 743-750. doi: 10.1016/ S1875-5364(15)30074-1 
Murdoch, C. E., Zhang, M., Cave, A. C., and Shah, A. M. (2006). NADPH oxidasedependent redox signalling in cardiac hypertrophy, remodelling and failure. Cardiovasc. Res. 71 (2), 208-215. doi: 10.1016/j.cardiores.2006.03.016

Musa, H., Kaur, K., O'Connell, R., Klos, M., Guerrero-Serna, G., Avula, U. M., et al. (2013). Inhibition of platelet-derived growth factor- $A B$ signaling prevents electromechanical remodeling of adult atrial myocytes that contact myofibroblasts. Heart Rhythm 10 (7), 1044-1051. doi: 10.1016/j. hrthm.2013.03.014

Nizamutdinova, I. T., Kim, Y. M., Jin, H., Son, K. H., Lee, J. H., Chang, K. C., et al. (2012). Tanshinone IIA inhibits TNF-alpha-mediated induction of VCAM-1 but not ICAM-1 through the regulation of GATA- 6 and IRF-1. Int. Immunopharmacol. 14 (4), 650-657. doi: 10.1016/j.intimp.2012.09.017

Owona, B. A., Njayou, N. F., Laufer, S. A., Schluesener, H. J., and Moundipa, P. F. (2013). Entada africana fraction $\mathrm{CH}(2) \mathrm{Cl}(2) / \mathrm{MEOH} 5 \%$ inhibits inducible nitric oxide synthase and pro-inflammatory cytokines gene expression induced by lipopolysaccharide in microglia. BMC Complement. Altern. Med. 13, 254. doi: 10.1186/1472-6882-13-254

Pan, Y., Qian, J. X., Lu, S. Q., Chen, J. W., Zhao, X. D., Jiang, Y., et al. (2017). Protective effects of tanshinone IIA sodium sulfonate on ischemia-reperfusioninduced myocardial injury in rats. Iran J. Basic Med. Sci. 20 (3), 308-315. doi: 10.22038/ijbms.2017.8361

Pello, A. M., Cristobal, C., Tarin, N., Huelmos, A., Acena, A., Carda, R., et al. (2015). Differential profile in inflammatory and mineral metabolism biomarkers in patients with ischemic heart disease without classical coronary risk factors. J. Cardiol. 66 (1), 22-27. doi: 10.1016/j.jjcc.2014.11.006

Price, R. L., Haley, S. T., Bullard, T. A., Goldsmith, E. C., Simpson, D. G., Thielen, T. E., et al. (2003). Effects of platelet-derived growth factor-AA and -BB on embryonic cardiac development. Anat. Rec. A Discov. Mol. Cell Evol. Biol. 272 (1), 424-433. doi: 10.1002/ar.a.10054

$\mathrm{Pu}$ J., Yuan, A., Shan, P., Gao, E., Wang, X., Wang, Y., et al. (2013). Cardiomyocyteexpressed farnesoid-X-receptor is a novel apoptosis mediator and contributes to myocardial ischaemia/reperfusion injury. Eur. Heart J. 34 (24), 1834-1845. doi: 10.1093/eurheartj/ehs011

Romson, J. L., Hook, B. G., Kunkel, S. L., Abrams, G. D., Schork, M. A., and Lucchesi, B. R. (1983). Reduction of the extent of ischemic myocardial injury by neutrophil depletion in the dog. Circulation 67 (5), 1016-1023. doi: 10.1161/01. CIR.67.5.1016

Simon, A. R., Takahashi, S., Severgnini, M., Fanburg, B. L., and Cochran, B. H. (2002). Role of the JAK-STAT pathway in PDGF-stimulated proliferation of human airway smooth muscle cells. Am J. Physiol. Lung Cell Mol. Physiol. 282 (6), L1296-L1304. doi: 10.1152/ajplung.00315.2001

Sun, Y. (2009). Myocardial repair/remodelling following infarction: roles of local factors. Cardiovasc. Res. 81 (3), 482-490. doi: 10.1093/cvr/cvn333

Thomas, W. G., Brandenburger, Y., Autelitano, D. J., Pham, T., Qian, H., and Hannan, R. D. (2002). Adenoviral-directed expression of the type $1 \mathrm{~A}$ angiotensin receptor promotes cardiomyocyte hypertrophy via transactivation of the epidermal growth factor receptor. Circ. Res. 90 (2), 135-142. doi: 10.1161/hh0202.104109

Tzanidis, A., Hannan, R. D., Thomas, W. G., Onan, D., Autelitano, D. J., See, F., et al. (2003). Direct actions of urotensin II on the heart: implications for cardiac fibrosis and hypertrophy. Circ. Res. 93 (3), 246-253. doi: 10.1161/01. RES.0000084382.64418.BC

Vantler, M., Karikkineth, B. C., Naito, H., Tiburcy, M., Didie, M., Nose, M., et al. (2010). PDGF-BB protects cardiomyocytes from apoptosis and improves contractile function of engineered heart tissue. J. Mol. Cell Cardiol. 48 (6), 1316-1323. doi: 10.1016/j.yjmcc.2010.03.008

Wagner, M. A., and Siddiqui, M. A. (2012). The JAK-STAT pathway in hypertrophic stress signaling and genomic stress response. JAKSTAT 1 (2), 131-141. doi: $10.4161 /$ jkst.20702

Wang, J., Hu, X., and Jiang, H. (2015a). HDAC inhibition: a novel therapeutic target for attenuating myocardial ischemia and reperfusion injury by reversing cardiac remodeling. Int. J. Cardiol. 190, 126-127. doi: 10.1016/j. ijcard.2015.04.172

Wang, J., Hu, X., and Jiang, H. (2015b). The Nrf-2/ARE-HO-1 axis: an important therapeutic approach for attenuating myocardial ischemia and reperfusion injury-induced cardiac remodeling. Int. J. Cardiol. 184, 263-264. doi: 10.1016/j. ijcard.2015.02.073
Wang, Y., Yan, X., Mi, S., Li, Z., Wang, Y., Zhu, H., et al. (2016). Naoxintong attenuates ischaemia/reperfusion injury through inhibiting NLRP3 inflammasome activation. J. Cell Mol. Med. 21 (1), 4-12. doi: 10.1111/jcmm.12915

Wang, Y. Z., Wharton, W., Garcia, R., Kraker, A., Jove, R., and Pledger, W. J. (2000). Activation of Stat3 preassembled with platelet-derived growth factor beta receptors requires Src kinase activity. Oncogene 19 (17), 2075-2085. doi: 10.1038/sj.onc. 1203548

Wei, B., Li, W. W., Ji, J., Hu, Q. H., and Ji, H. (2014). The cardioprotective effect of sodium tanshinone IIA sulfonate and the optimizing of therapeutic time window in myocardial ischemia/reperfusion injury in rats. Atherosclerosis 235 (2), 318-327. doi: 10.1016/j.atherosclerosis.2014.05.924

Wincewicz, A., and Sulkowski, S. (2017). Stat proteins as intracellular regulators of resistance to myocardial injury in the context of cardiac remodeling and targeting for therapy. Adv. Clin. Exp. Med. 26 (4), 703-708. doi: 10.17219/ acem/62693

Wu, Q., Wang, W., Li, S., Nagarkatti, P., Nagarkatti, M., Windust, A., et al. (2012). American ginseng inhibits vascular smooth muscle cell proliferation via suppressing Jak/Stat pathway. J. Ethnopharmacol. 144 (3), 782-785. doi: 10.1016/j.jep.2012.09.046

Wu, W. Y., Wang, W. Y., Ma, Y. L., Yan, H., Wang, X. B., Qin, Y. L., et al. (2013). Sodium tanshinone IIA silate inhibits oxygen-glucose deprivation/ recovery-induced cardiomyocyte apoptosis via suppression of the NF-kappaB/TNF-alpha pathway. Br. J. Pharmacol. 169 (5), 1058-1071. doi: 10.1111/bph.12185

Yan, X., Anzai, A., Katsumata, Y., Matsuhashi, T., Ito, K., Endo, J., et al. (2013). Temporal dynamics of cardiac immune cell accumulation following acute myocardial infarction. J. Mol. Cell Cardiol. 62, 24-35. doi: 10.1016/j. yjmcc.2013.04.023

Yang, M., Orgah, J., Zhu, J., Fan, G., Han, J., Wang, X., et al. (2016). Danhong injection attenuates cardiac injury induced by ischemic and reperfused neuronal cells through regulating arginine vasopressin expression and secretion. Brain Res. 1642, 516-523. doi: 10.1016/j.brainres.2016.04.046

Yellon, D. M., and Hausenloy, D. J. (2007). Myocardial reperfusion injury. N. Engl. J. Med. 357 (11), 1121-1135. doi: 10.1056/NEJMra071667

Zhang, L., Ma, J., Shen, T., Wang, S., Ma, C., Liu, Y., et al. (2012). Platelet-derived growth factor (PDGF) induces pulmonary vascular remodeling through 15-LO/15-HETE pathway under hypoxic condition. Cell Signal. 24 (10), 19311939. doi: 10.1016/j.cellsig.2012.06.007

Zhang, M. Q., Zheng, Y. L., Chen, H., Tu, J. F., Shen, Y., Guo, J. P., et al. (2013). Sodium tanshinone IIA sulfonate protects rat myocardium against ischemiareperfusion injury via activation of PI3K/Akt/FOXO3A/Bim pathway. Acta Pharmacol. Sin. 34 (11), 1386-1396. doi: 10.1038/aps.2013.91

Zhang, Y., Wei, L., Sun, D., Cao, F., Gao, H., Zhao, L., et al. (2010). Tanshinone IIA pretreatment protects myocardium against ischaemia/reperfusion injury through the phosphatidylinositol 3-kinase/Akt-dependent pathway in diabetic rats. Diabetes Obes. Metab. 12 (4), 316-322. doi: 10.1111/ j.1463-1326.2009.01166.x

Zhao, W., Zhao, T., Huang, V., Chen, Y., Ahokas, R. A., and Sun, Y. (2011). Platelet-derived growth factor involvement in myocardial remodeling following infarction. J. Mol. Cell Cardiol. 51 (5), 830-838. doi: 10.1016/j. yjmcc.2011.06.023

Zhou, G., Jiang, W., Zhao, Y., Ma, G., Xin, W., Yin, J., et al. (2003). Sodium tanshinone IIA sulfonate mediates electron transfer reaction in rat heart mitochondria. Biochem. Pharmacol. 65 (1), 51-57. doi: 10.1016/S0006-2952(02)01447-8

Conflict of Interest: The authors declare that the research was conducted in the absence of any commercial or financial relationships that could be construed as a potential conflict of interest.

Copyright (c) 2019 Liu, Zhou, Meng, Huang, Liu, Tie, Zhuang, Chen, Zhang, Wei, Huang, Li, Wang, Fan and Liu. This is an open-access article distributed under the terms of the Creative Commons Attribution License (CC BY). The use, distribution or reproduction in other forums is permitted, provided the original author(s) and the copyright owner(s) are credited and that the original publication in this journal is cited, in accordance with accepted academic practice. No use, distribution or reproduction is permitted which does not comply with these terms. 